\title{
AN ASSESSMENT OF THE LOSS DISTRIBUTION IN FRANCIS TURBINES
}

\author{
R. SUZUKI, Y. QIAN \\ Fuji Electric Co., Ltd. \\ 1-1, Tanabeshinden, Kawasaki-ku, Kawasaki, 210 JAPAN \\ T. KITAHORA, J. KUROKAWA \\ Yokohama National University \\ 156 Tokiwadai, Hodogaya-ku, Yokohama, 240 JAPAN
}

\begin{abstract}
Scalable loss in Francis model turbine is analyzed for four runners with various number of blades which are tested in the same model turbine. The flow in the stay vane, the guide vane and the runners are analyzed by a 3D Euler flow analysis code, and then three methods of boundary layer calculations are carried out in order to obtain scalable loss. As for spiral casing and draft tube, scalable loss is obtained by the analogy of pipe flow.

From the comparison between runners, the difference in peak efficiency seems to be mainly caused by non-scalable losses. Therefore scalable loss itself is most suitable to be prescribed in the scale-up formula than loss distribution coefficient.

Problems on the effect of number of blades might be solved by using scalable loss in scale-up formulae.
\end{abstract}

\section{Introduction}

The efficiency performance of prototype turbines is predicted by applying so-called scaleup formulae to the performance of model turbines. Some famous formulae, for example Moody, Ackeret and Hutton formulae, had been used for many years for this purpose. Recently the study on the scale effect has been activated, and some new methods of scale- 
up were proposed. Japan Society of Mechanical Engineers (JSME) established a standard based on numerical loss analyses and including the effect of wall roughness. IEC established a new standard based on the strict consideration on the step-up to a hydraulically smooth prototype emphasizing the effect of test Reynolds number of model turbines. Both in JSME and IEC the loss distribution coefficient $V$ was used. Spurk/Grein, starting from dimensional analysis, introduced a formula using the efficiency at infinite Reynolds number. Nichtawitz claimed it was not reasonable that a model turbine with lower peak efficiency would be rewarded with a higher step-up. Ida and Nichtawitz proposed new formulae in order to avoid this absurdity. They introduced $\delta_{\text {ref }}$ in their formulae.

From the viewpoint of the value to be prescribed, there are three standpoints;

(1) To prescribe loss distribution coefficient

Ackeret, Hutton, JSME and IEC formulae belong to this group, and they are based on the idea that the ratio of scalable (frictional) loss to the total hydraulic loss would be almost constant for various turbines.

(2) To prescribe non-scalable loss

The proposal of Spurk/Grein corresponds to this standpoint if $\eta_{\infty}$ is to be accumulated experimentally. It is based on the idea that non-scalable loss is almost constant for various turbines. In other words, the difference in peak efficiency is mainly caused by scalable loss.

(3) To prescribe scalable loss

Ida and Nichtawitz formulae belong to this group, and they are based on the idea that the difference in peak efficiency is mainly caused by non-scalable loss.

All the ideas about the cause of difference in peak efficiency mentioned above are, of course, idealized ones. But it is necessary to clarify which is most realistic.

On the other hand, there was a discussion in Europe that turbines with large number of runner blades would gain high efficiency step-up because of high ratio of scalable loss. This claim has never been adopted in any standard and $V$ or other values are prescribed constant regardless of the number of runner blades. Even which formula is to be adopted, it is better to be discussed how the number of blade affect to efficiency step-up.

The purpose of this paper is to present some data for judging the key factor of the difference in peak efficiencies, and also the effect of the number of runner blades.

\section{Specified Model Turbine}

The specifications of Francis turbine used for the investigation is listed in Table 2. Spe- 
TABLE 1. Scale-up Formulae

\begin{tabular}{|c|c|c|}
\hline Proposer & Formula & \\
\hline $\begin{array}{l}\text { JSME S008 } \\
\text { (1989) }\end{array}$ & $\begin{array}{c}\frac{1-\eta_{E P}}{1-\eta_{E M}}=\frac{\eta_{E P}}{\eta_{E M}}\{(1-V)+V \Lambda\} \\
\Lambda=\left(D_{P} / D_{M}\right)^{0.18}\left\{(1-\beta)+\beta\left(e_{P} / e_{M}\right)^{0.16}\right\}\end{array}$ & $\begin{array}{l}\text { (1) } \\
(2)\end{array}$ \\
\hline \multirow[t]{2}{*}{$\begin{array}{l}\text { IEC } 995 \\
(1991)\end{array}$} & $\Delta \eta_{h}=\delta_{\text {ref }}\left[\left(\frac{R e_{u \text { ref }}}{R e_{u} \mathrm{M}}\right)^{0.16}-\left(\frac{R e_{u \text { ref }}}{R e_{u \mathrm{P}}}\right)^{0.16}\right]$ & (3) \\
\hline & $\delta_{\text {ref }}=\frac{1-\eta_{h o p t ~}}{\left(\frac{R e_{u \text { ref }}}{R e_{u \text { opt } \mathrm{M}}}\right)^{0.16}-\frac{1-V_{\text {ref }}}{V_{\text {ref }}}}$ & (4) \\
\hline $\begin{array}{l}\text { Spurk/Grein } \\
\text { (1992) }\end{array}$ & $\Delta \eta_{h}=\left(\eta_{h \infty}-\eta_{h \mathrm{M}}\right)\left[1-\left(\frac{\operatorname{Re}_{\mathrm{M}}}{\operatorname{ReP}_{\mathrm{P}}}\right)^{\alpha}\right]$ & (5) \\
\hline \multirow[t]{2}{*}{$\begin{array}{c}\text { Ida } \\
(1993)\end{array}$} & $\frac{\eta_{E P}}{\eta_{E M}}=\frac{1}{1-\delta_{E}\left(1-\Lambda^{\prime}\right)}$ & (6) \\
\hline & $\Lambda=\left(D_{\mathrm{P}} / D_{\mathrm{M}}\right)^{-0.18}\left\{(1-\beta)+\beta\left(e_{\mathrm{P}} / e_{\mathrm{M}}\right)^{0.18}\right\}$ & (7) \\
\hline $\begin{array}{l}\text { Nichtawitz } \\
\text { (1994) }\end{array}$ & $\Delta \eta_{h}=S_{0}\left(\frac{R e_{0}}{R e_{M}}\right)^{n}-\left(\frac{R e_{0}}{R e_{\mathrm{P}}}\right)^{n}$ & (8) \\
\hline
\end{tabular}

Note; Some formulae include the conversion of operating condition as well as the efficiency step-up procedure for pumping operation. But only the efficiency step-up formulae for turbine are contrasted here.

$\eta_{E}$ in the formulae by JSME and Ida is the specific energy efficiency, the ratio of the specific hydraulic energy available for the runner to the specific hydraulic energy of the machine (see equations (9) and (10) below).

cific speed $n_{s Q}=n Q^{0.5} / H^{0.75}$ is around $40\left(\mathrm{~min}^{-1}, \mathrm{~m}^{3} / \mathrm{s}, \mathrm{m}\right)$. Runners $\mathrm{A}$ and $\mathrm{B}$ have the same number of blades but Runner $A$ shows higher efficiency. Runners $C$ and $D$ have smaller number of blades, and shows lower efficiency than Runners $A$ and $B$. Here, $\eta_{E}$ denotes specific energy efficiency;

$$
\eta_{E}=E_{m} / E=1-E_{L 12} / E
$$




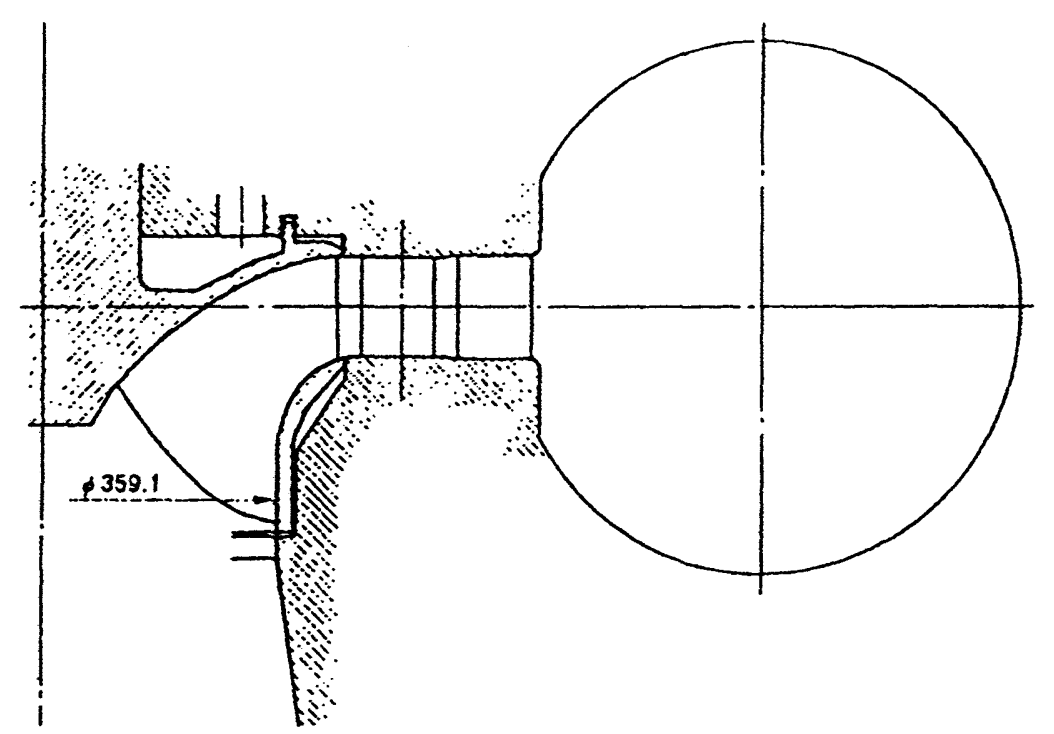

FIGURE 1. Sectional View of the Specified Model Turbine

TABLE 2. Specifications of Runners

\begin{tabular}{c|c|c|c|c}
\hline Runner & $\mathrm{A}$ & $\mathrm{B}$ & $\mathrm{C}$ & $\mathrm{D}$ \\
\hline$D(\mathrm{~mm})$ & 359.1 & 359.1 & 359.1 & 359.1 \\
$R e$ & $6.3 \times 10^{6}$ & $6.3 \times 10^{6}$ & $6.3 \times 10^{6}$ & $6.3 \times 10^{6}$ \\
$n_{S Q_{\text {opl }}}$ & 40.4 & 38.3 & 37.0 & 35.6 \\
\hline$n_{E D} / n_{E D(\mathrm{~A})}$ & 1.00 & 1.00 & 1.00 & 1.00 \\
$Q_{E D} / Q_{E D(A)}$ & 1.00 & 1.00 & 0.963 & 0.969 \\
\hline$n_{E D} / n_{E D_{\text {op }}}$ & 1.00 & 1.03 & 1.00 & 1.05 \\
$Q_{E D} / Q_{E D o p 1}$ & 1.00 & 1.04 & 1.15 & 1.12 \\
\hline$\eta_{E}(\%)$ & 95.0 & 93.6 & 92.0 & 91.3 \\
$\eta_{E o p 1}(\%)$ & 95.0 & 93.7 & 92.4 & 91.9 \\
\hline$Z_{R}$ & 13 & 13 & 9 & 7 \\
\hline
\end{tabular}

where $E_{m^{\prime}} E_{L / 2}$ and $E$ indicate specific hydraulic energy of the runner, specific energy loss of the machine and specific hydraulic energy of the machine respectively. Therefore;

$$
\eta_{E}=\eta_{h} / \eta_{Q} \eta_{R}
$$

where $\eta_{h}, \eta_{Q}$ and $\eta_{R}$ are hydraulic (internal) efficiency, discharge (volumetric) efficiency and power efficiency (related to disk friction) respectively.

The values $n_{E D}$ and $Q_{E D}$ listed in Table 2 without subscript are the speed factors and discharge factors of the operating conditions analyzed. Those with subscript (A) indicate the values for Runner $A$, and subscripts opt denotes the best efficiency point of each runner. Runner $A$ is analyzed at the best efficiency point. Analyses for other runners are carried 
out at the same guide vane opening and the same speed factor as Runner $\mathrm{A}$. The discrepancy between the analyzed point and the best efficiency point for each runner is not so large, and the maximum difference in efficiency is $0.6 \%$ in Runner $D$.

\section{Numerical Analysis of Scalable Loss}

\subsection{INTERNAL FLOW ANALYSES IN CASCADES}

The flow in stay vanes, guide vanes and runner blades are analyzed with 3D Euler code with pseudo-compressibility and implicit formulation of finite difference. This Euler code has the same numerical schemes as the Navier-Stokes code [6], but the effect of viscosity is neglected and the boundary condition on the wall surfaces is different. Figure 2 shows the flow field for Runner A obtained from the analysis.

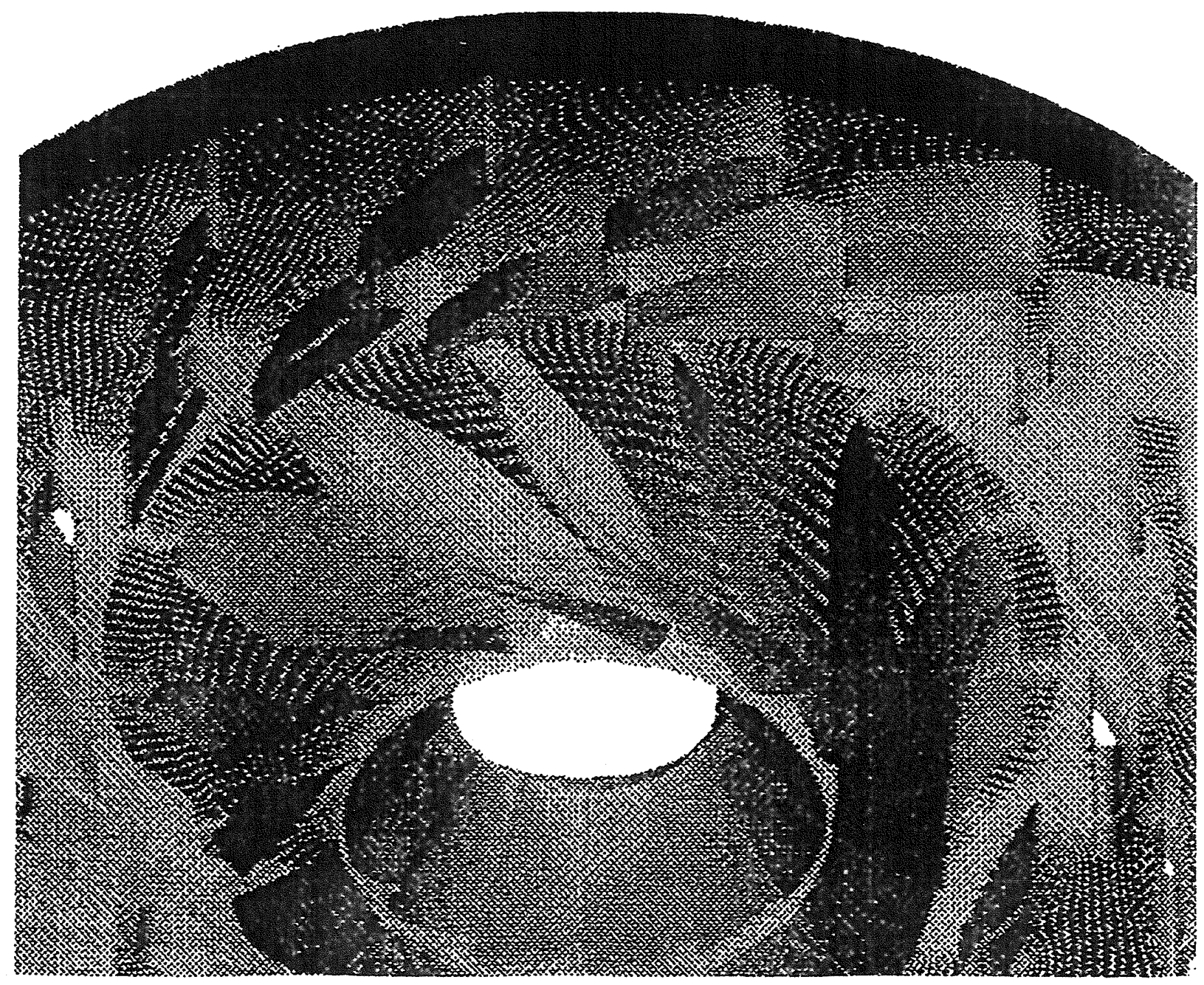

FIGURE 2. Flow Velocity Vectors in Cascades 


\subsection{CALCULATION OF SCALABLE LOSS}

\subsubsection{Scalable Loss in Runner}

Three Dimensional Boundary Layer Analysis. Three dimensional boundary layer calculated on the wall surface in runner using the velocity distribution obtained by 3 Euler flow analysis. Coriolis force and centrifugal force are considered. The numeric scheme of calculation is described in [7]. Figure 3 illustrates the result of calculation $f$ Runner A.

The scalable loss is obtained from the energy thickness of boundary layer at the outlet blade surfaces and of crown and band surfaces.

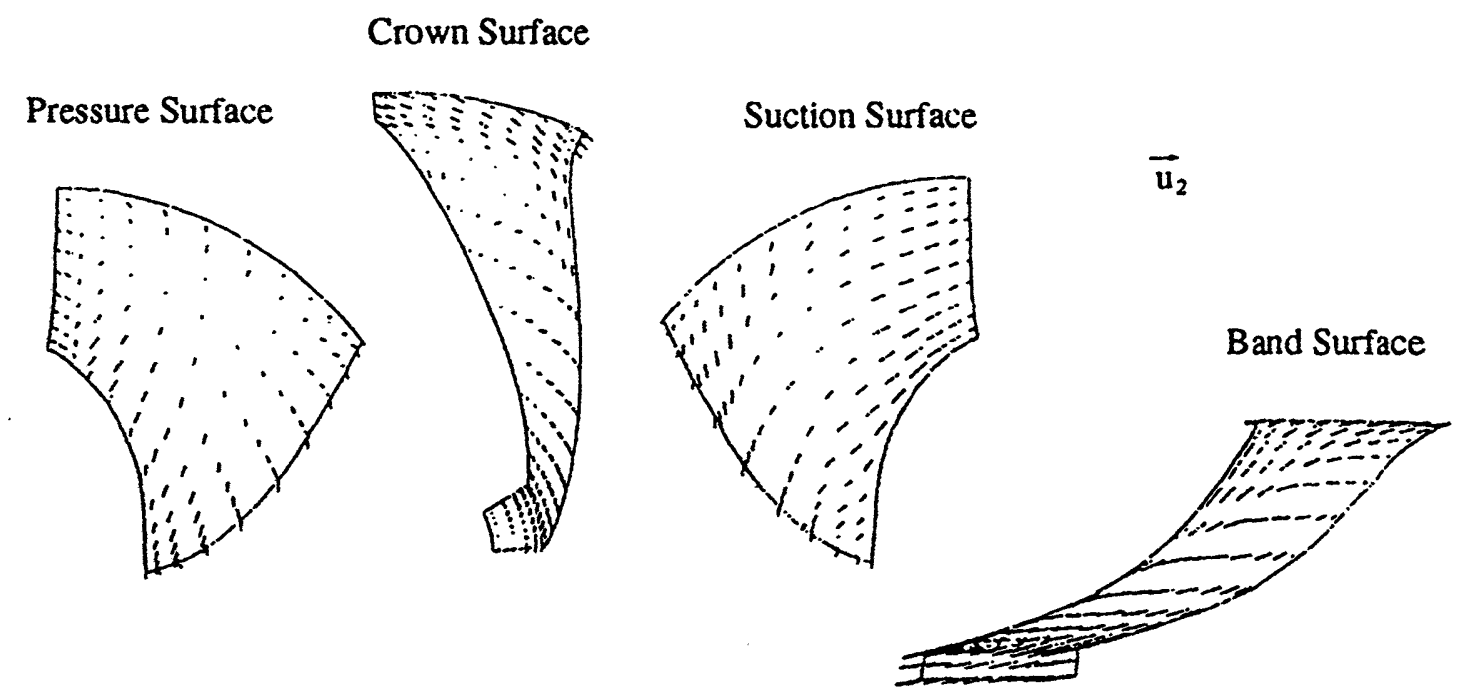

(a) Main Flow Velocity on the Walls

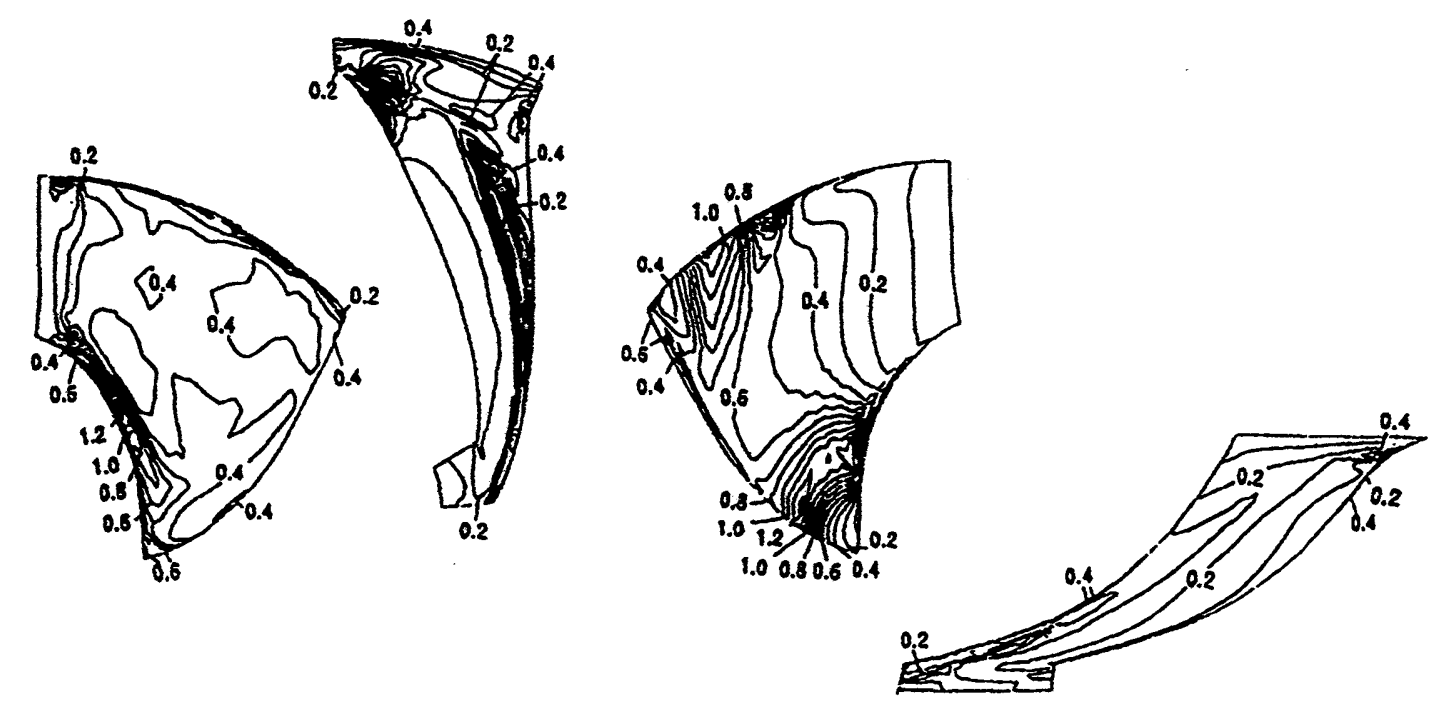

(b) Boundary Layer Thicness $\delta / \mathrm{D}_{2}(\%)$

FIGURE 3. Three Dimensional Boundary Layer on Runner Surface 
One Dimensional Boundary Layer Calculation. One dimensional approximate boundary layer calculation is made along the grid lines using the velocity distribution obtained by 3D Euler flow analysis. Coriolis force and centrifugal force are not considered. The discrepancy in flow direction from the grid lines are also neglected.

The scalable loss is obtained from the energy thickness of boundary layer at the outlet of blade surfaces and of crown and band surfaces.

Integration of Shearing Stress. The scalable'loss is approximately estimated integrating the local shearing stress of a flat plate, which is obtained from the local velocity and the distance from the inlet. The velocity distribution on the wall surfaces obtained from 3D Euler flow analysis is used.

\subsubsection{Scalable Loss in Stay Vanes and Guide Vanes}

The scalable losses in stay vanes and guide vanes are obtained only by the integration of shearing stress mentioned above, because the flow distortion is not so large.

\subsubsection{Scalable Loss in Spiral Casing and Draft Tube}

The scalable losses in spiral casing and draft tube are calculated with Colebrook's equation by analogy of pipe friction.

\section{Results and Discussions}

Table 3 shows the results of scalable loss analyses. $\delta_{E}$ denotes the total scalable deficiency (relative scalable loss of specific hydraulic energy). $\delta_{E S}, \delta_{E R}$ and $\delta_{E D}$ are the scalable deficiencies in the upstream parts of runner (spiral casing to guide vanes), in the runner and in the draft tube respectively. $\delta_{E n s}$ denotes the non-scalable deficiency which is obtained subtracting $\delta_{E}$ from the total specific hydraulic loss $E_{L 12}=100-\eta_{E}$. The loss distribution coefficients are also tabulated. The subscripts $S, R$ and $D$ correspond to those of scalable deficiencies. Three values in array divided by slash marks correspond to the methods of scalable loss calculation.

Figure 4 illustrates the variation of the total scalable deficiency. Three kinds of bars correspond to the loss calculation methods. In spite of large difference in efficiency among four runners, the scalable deficiency is almost constant.

Figure 5 shows the variation of the non-scalable deficiency. $\delta_{E n s}$ steeply increases from Runner $A$ to $D$, indicating that the discrepancy in efficiency is mainly caused by non- 
TABLE 3. Scalable and Non-scalable Deficiencies

\begin{tabular}{c|c|c|c|c}
\hline Runner & $\mathrm{A}$ & $\mathrm{B}$ & $\mathrm{C}$ & $\mathrm{D}$ \\
\hline$\eta_{E}$ & 95.04 & 93.60 & 91.97 & 91.25 \\
\hline$\delta_{E S}(\%)$ & 1.75 & 1.75 & 1.65 & 1.64 \\
$\delta_{E R}(\%)$ & $0.71 / 0.96 / 1.00$ & $1.01 / 1.49 / 1.20$ & $1.24 / 1.72 / 1.40$ & $1.26 / 1.67 / 1.35$ \\
$\delta_{E D}(\%)$ & 0.04 & 0.04 & 0.04 & 0.04 \\
\hline$\delta_{E}(\%)$ & $2.50 / 2.76 / 2.79$ & $2.80 / 3.28 / 2.99$ & $2.91 / 3.39 / 3.07$ & $2.95 / 3.35 / 3.04$ \\
\hline$\delta_{E_{n s}}(\%)$ & $2.46 / 2.20 / 2.17$ & $3.60 / 3.12 / 3.41$ & $5.12 / 4.64 / 4.96$ & $5.80 / 5.40 / 5.71$ \\
\hline$V_{S}$ & 0.35 & 0.27 & 0.20 & 0.19 \\
$V_{R}$ & $0.14 / 0.19 / 0.20$ & $0.16 / 0.23 / 0.19$ & $0.16 / 0.21 / 0.17$ & $0.14 / 0.19 / 0.16$ \\
$V_{D}$ & 0.01 & 0.01 & 0.01 & 0.01 \\
\hline$V$ & $0.50 / 0.56 / 0.56$ & $0.44 / 0.51 / 0.47$ & $0.36 / 0.42 / 0.38$ & $0.34 / 0.38 / 0.35$ \\
\hline
\end{tabular}

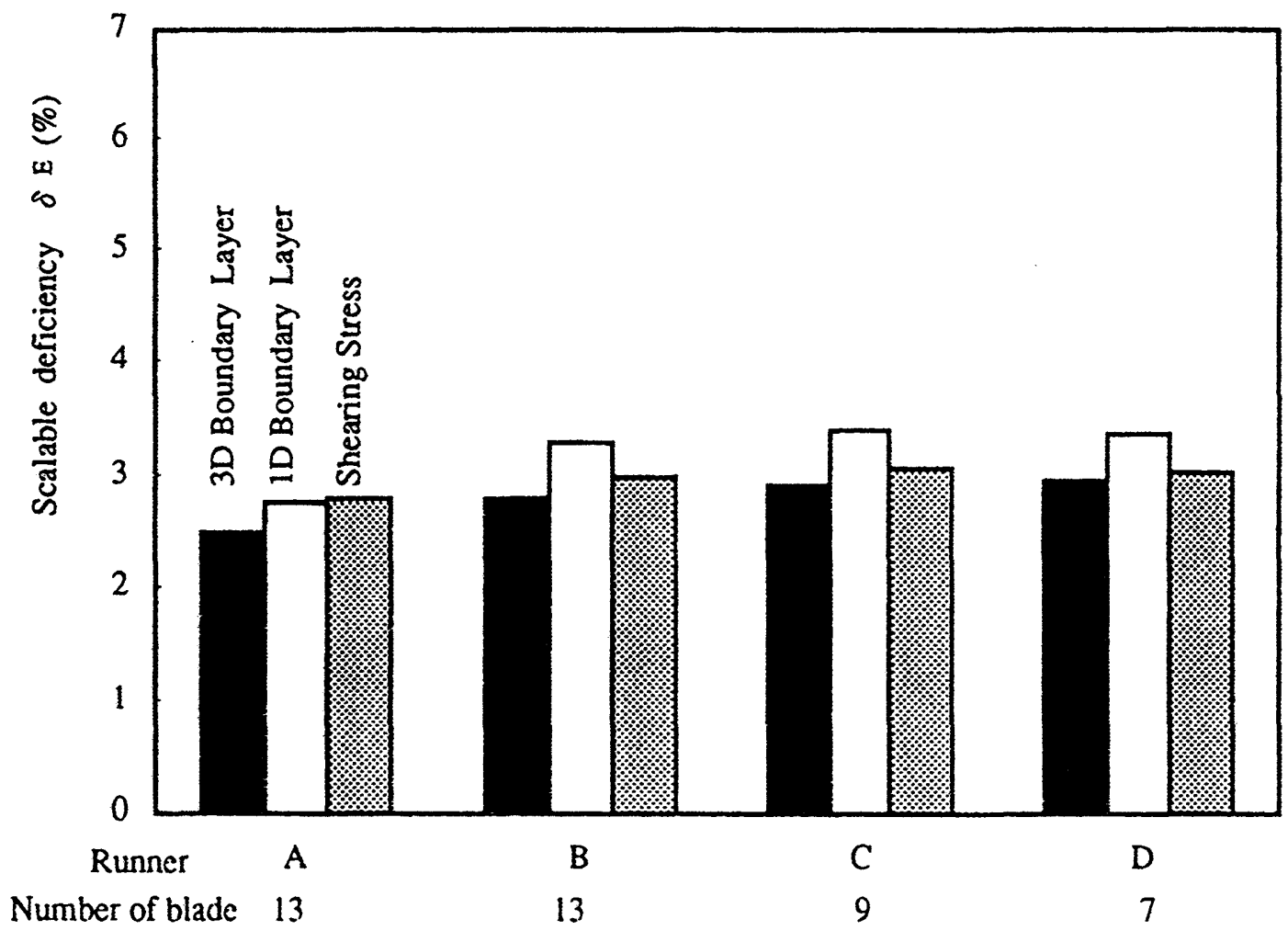

FIGURE 4. Scalable Deficiency

scalable loss. From Figure 6 illustrating the variation of the loss distribution coefficient, the same conclusion can be drawn.

Changing the point of view to the effect of the number of blades, the loss distribution coefficient decreases apparently with decreasing number of blades. If this result is extrapolated toward larger number of blades, it would be concluded that the increase in the number of blades would increase the value of $V$. 


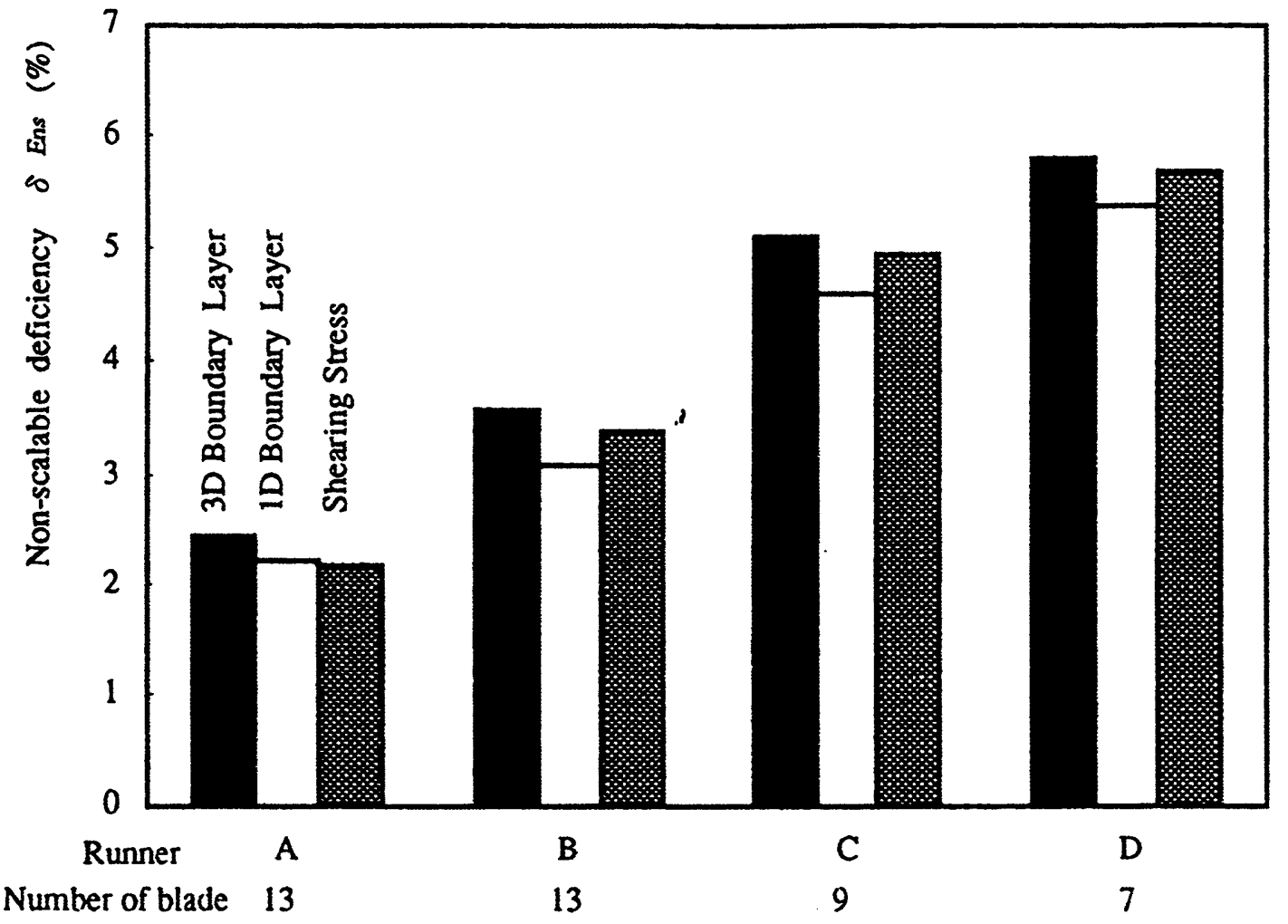

FIGURE 5. Non-scalable Deficiency

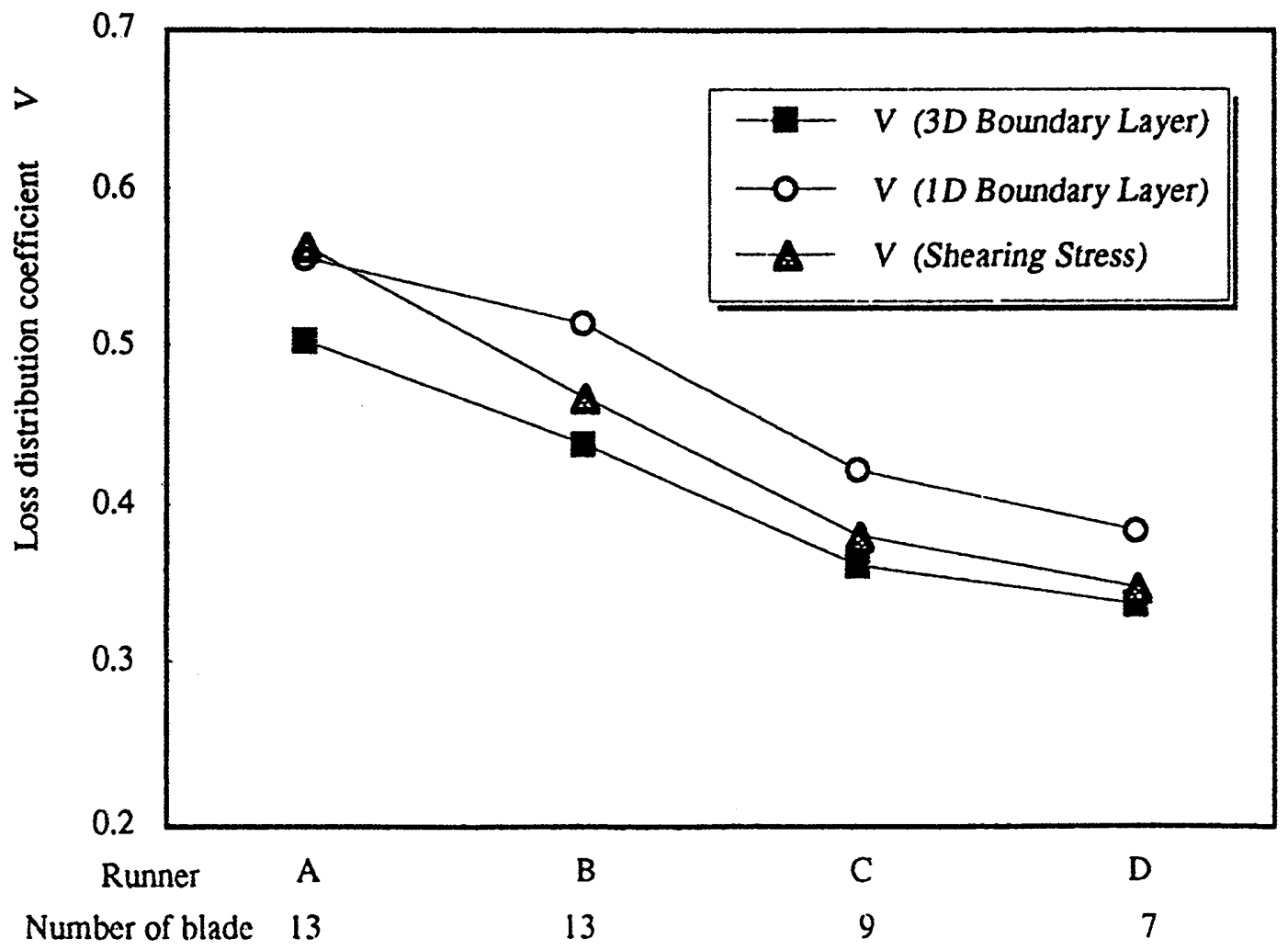

FIGURE 6. Loss Distribution Coefficient 
Irrespective of the above mentioned tendency in loss distribution coefficient, however, the discussion about the effect of the number of blades might be disposed of when scalable loss is prescribed in scale-up formulae.

\section{Conclusion}

Results of scalable loss analyses for four Francis tunners tested in the same model turbine are presented.

Analyzed results showed best regularity in scalable loss. Non-scalable loss steeply increased and loss distribution coefficient considerably decreased in proportion as efficiency dropped. Then lower peak efficiency seems to be mainly caused by non-scalable loss. This means that scalable loss is most suitable for prescription in scale-up procedures. Although the possibility could be seen on the increase in loss distribution coefficient with increasing number of blades, yet the problem would be solved by prescribing scalable loss instead of loss distribution coefficient.

\section{Acknowledgement}

This study was strongly motivated by the activities of the committee on preparing the revised JSME S008. The 3D boundary layer analyses are performed by Mr. Matsumoto, the graduate student in Yokohama National University.

\section{References}

1. JSME Standard S008, Performance Conversion Method for Hydraulic Turbines and Pumps (1989).

2. IEC Publication 995, Determination of the Prototype Performance from Model Acceptance Tests of Hydraulic Machines with Consideration of Scale Effects (1991).

3. Spurk, J.H. and Grein, H., Performance Predictions of Hydraulic Machines by Dimensional Considerations, Water Power \& Dam Construction (1993-11), 42-49.

4. Ida, T., New Formula for Efficiency Step-up of Hydraulic Turbines, Proc. 17th IAHR Symposium (Beijing, 1994), 827-840.

5. Nichtawitz, A., Discussion on Step-up Procedures in Hydraulic Machines, Proc. 17th IAHR Symposium (Beijing, 1994), 841-852.

6. Qian, Y., Arakawa, C., Kubota, T. and Suzuki, R., Numerical Flow Simulation on Channel Vortex in Francis Runner, Proc. 17th IAHR Symposium (Beijing, 1994), 237-247.

7. Kurokawa, J., Kitahora, T., et al., Prediction of Scalable Loss in Francis Runners of Different Specific Specd, Proc. 18th IAHR Symposium (Valencia, 1996). 